\title{
Errata
}

\section{A closed-form solution for the stress field in a rigid- perfectly plastic material under plane-strain conditions}

Acta Mechanica 77, 307-313 (1989)

D. E. Panayotounakos and N. P. Andrianopoulos, Athens, Greece

Trying to expand the closed-form solution of the partial differential equation (P.D.E.), Eq. (2.13), described in the above referenced paper, we detected an unhappy algebraic error affecting Section 3 , and consequently the respective results. This error is located in deriving Eq. (3.8), which is obtained by combining Eqs. (3.3) and (3.4) and not from Eq. (3.4) only. Thus, the set of Monge's equations consists of Eqs. (3.5), (3.8), and (3.7). This latter equation was not taken into consideration in presenting the solution. Therefore, the procedure described in $\S 3$ of the above referenced paper must be corrected as follows.

\section{Closed-form solutions of Eq. (2.13)}

The nonlinear second order P.D.E. given by (2.13) can be expressed in the form of the Monge equation [6]

$R r+S s+T t=V$

where

$R=-T=1 ; \quad S= \pm \frac{2 z}{\left(\varkappa^{2}-z^{2}\right)^{1 / 2}} ; \quad V=\mp \frac{2 x^{2}}{\left(\varkappa^{2}-z^{2}\right)^{3 / 2}} p q$.

The set of Monge's equations which are equivalent to Eqs. (3.2) is

$d p d y-d q d x=\omega_{1} p q d x d y$,

$d y^{2}-d x^{2}=\omega_{2} d x d y$,

$d z=p d x+q d y$,

in which

$\omega_{1}=\mp 2 x^{2} /\left(x^{2}-z^{2}\right)^{3 / 2} ; \quad \omega_{2}= \pm 2 z /\left(x^{2}-z^{2}\right)^{1 / 2}$.

We may find solutions of Eq. (2.13) of the form

$p=c q, \quad c=$ constant 
Solving Eq. (3.4) for $(d y / d x)$ we deduce

$d y / d x=\Omega ; \quad \Omega=\left[\omega_{2} \pm\left(\omega_{2}{ }^{2}+4\right)^{1 / 2}\right] / 2$.

Combining Eq. (3.3) together with (3.7) and (3.8) we derive

$(c \Omega-1) d q=\omega_{1} c q^{2} \Omega d x$.

By means of (3.8) and (3.9) we evaluate

$d x=(c \Omega-1) d q / \omega_{1} c q^{2} \Omega ; \quad d y=(c \Omega-1) d q / \omega_{1} c q^{2}$.

Therefore, using (3.7) and (3.10), the third of Monge's equations furnishes

$\frac{\omega_{1} c \Omega}{(c \Omega-1)(\Omega+c)} d z=\frac{d q}{q}$.

Since

$\omega_{1} c \Omega /(c \Omega-1)(\Omega+c)=\omega_{1} c /\left(c^{2}+c \omega_{2}-1\right)$,

and taking into account relations (3.6), the integration of Eq. (3.11) results in

$q=\lambda /\left(c^{2}+c \omega_{2}-1\right)$,

where $\lambda$ is a new constant of integration.

From now on, by means of (3.13) and (3.7), Eq. (3.5) becomes

$\left(c^{2}+c \omega_{2}-1\right) d z=\lambda(c d x+d y)$

the integration of which, by way of (3.6), results in

$\left(c^{2}-1\right) z \mp 2 c\left(x^{2}-z^{2}\right)^{1 / 2}=\lambda(c x+y)+\mu$,

where $\mu$ is a third constant of integration.

Equation (3.15) constitutes a closed-form solution of the nonlinear P.D.E. (2.13) and represents a three-parameters family of surfaces of the form

$f\left(x, y, \tau_{x y} ; c, \lambda, \mu\right)=0$,

which, further being solved for $\tau_{x y}$, furnishes the shear stress-resultant as a complete second order algebraic equation for the $x, y$ variables and the $c, \lambda, \mu$-parameters, namely:

$\tau_{x y}=\left\{A x+B y+C \pm\left[\left(A^{*} x+B^{*} y+C^{*}\right)^{2}+D^{2}\right]^{1 / 2}\right\} / E^{2}=F_{1}(x, y)$

in which

$A=\left(c^{2}-1\right) c \lambda ; \quad B=\left(c^{2}-1\right) \lambda ; \quad C=\left(c^{2}-1\right) \mu ; \quad D^{2}=4 \varkappa^{2} c^{2}\left(c^{2}+1\right)^{2}, \quad E=c^{2}+1 ;$

$A^{*}=2 C^{2} \lambda ; \quad B^{*}=2 C \lambda ; \quad C^{*}=2 C \mu$.

Taking into account the equilibrium differential equations, as well as the solution (3.17), we evaluate the normal stress resultants as follows:

$\sigma_{x}=F_{2}(x, y)+f(y) ; \quad \sigma_{y}=F_{3}(x, y)+g(x)$, 
where

$F_{2}(x, y)=-\frac{1}{E^{2}}\left\{B x \pm \frac{B^{*}}{A^{*}}\left[-\left(A^{*} x+B^{*} y+C^{*}\right)^{2}+D^{2}\right]^{1 / 2}\right\}$
$F_{3}(x, y)=-\frac{1}{E^{2}}\left\{A y \pm \frac{A^{*}}{B^{*}}\left[-\left(A^{*} x+B^{*} y+C^{*}\right)^{2}+D^{2}\right]^{1 / 2}\right\}$

while $f(y), g(x)$ are arbitrary. Furthermore, since at any point of the body the von Mises-Hencky condition must be valid, we conclude that

$f(y)= \pm 2\left[x^{2}-F_{1}^{2}(x, y)\right]^{1 / 2}-F_{2}(x, y)+F_{3}(x, y)+g(x)$.

Consequently, the stress-resultants can be written in the final form

$\tau_{x y}=F_{1}(x, y) ; \quad \sigma_{x}= \pm 2\left[x^{2}-F_{1}^{2}(x, y)\right]^{1 / 2}+F_{3}(x, y)+g(x) ; \quad \sigma_{y}=F_{3}(x, y)+g(x)$

in which $F_{1}$ and $F_{3}$ are given in Eqs. (3.17) and (3.18).

\section{The beneficial influence}

\section{of matrix anisotropy in fiber composites}

Acta Mechanica 97, 127-139 (1993)

P. S. Theocaris, Athens, Greece

Relation (1) should read as follows:

$K_{T}=1=\left\{2\left(\frac{E_{L}}{E_{T}}\right)^{1 / 2}+2\left(\frac{E_{L}}{2 G_{L}}-v_{L}\right)\right\}^{1 / 2} \frac{b}{a}$

Verleger: Springer-Verlag KG, Sachsenplatz 4-6, A-1201 Wien. - Herausgeber: Prof, Dipl-Ing. Dr. Hans Troger und Prof. Dipl-Ing. Dr. Franz Ziegler, beide Technische Universität, Karlsplatz 13, A-1040 Wien. - Redaktion: Karlsplatz 13, A-1040 Wien. - Hersteller: Druckerei zu Altenburg GmbH, D-04600 Altenburg - Verlagsort: Wien. - Hersiellungsort: Altenburg. - Printed in Germany. 